\title{
DIFFERENTIAL EQUATIONS AND THE BETHE ANSATZ
}

\author{
PATRICK DOREY \\ Department of Mathematical Sciences, \\ University of Durham, \\ Durham DH1 3LE, UK. \\ p.e.dorey@durham.ac.uk \\ CLARE DUNNING \\ Department of Mathematics, \\ University of York, \\ York YO1 5DD, UK. \\ tcd1@york.ac.uk \\ ADAM MILLICAN-SLATER \\ Department of Mathematical Sciences, \\ University of Durham, \\ Durham DH1 3LE, UK. \\ adam.millican-slater@durham.ac.uk \\ ROBERTO TATEO \\ Department of Mathematical Sciences, \\ University of Durham, \\ Durham DH1 3LE, UK.* \\ roberto.tateo@durham.ac.uk
}

\begin{abstract}
We review some surprising links which have been discovered in the last few years between the theory of certain ordinary differential equations, and particular integrable lattice models and quantum field theories in two dimensions. An application of this correspondence to a problem in non-Hermitian (PT-symmetric) quantum mechanics is also discussed.

Contribution to the proceedings of the International Congress of Mathematical Physics, Lisbon 2003; preprint DCPT-03/45, hep-th/0309054.
\end{abstract}

\section{Introduction}

In the last few years, detailed connections have begun to emerge between two previouslyseparated areas of mathematical physics: the spectral properties of ordinary differential equations such as the Schrödinger equation, and the study of integrable lattice models and integrable quantum field theories using techniques related to the Bethe ansatz. This is

*Address after November 2003: Dipartimento di Fisica Teorica, Università di Torino, Via P. Giuria 1, 10125 Torino, Italy. 
sometimes given the (perhaps over-grand) title of the 'ODE/IM correspondence'. While the correspondence remains at the level of a mathematical coincidence, albeit a detailed one, it has already provided some important new insights into a variety of problems.

On the 'ODE' (ordinary differential equations) side, some of the key themes are Hermitian and non-Hermitian spectral problems, Schrödinger equations, the WKB method, analytic continuation and $\mathcal{P} \mathcal{T}$-symmetry. These have been developed by a variety of authors; some important names and references for the story that we want to tell are Sibuya [1], Voros [2], Bessis and Zinn-Justin [3], and Bender and Boettcher [4,5].

The 'IM' (integrable models) aspect has a similarly long history. The theories relevant for the first example of the correspondence to be discovered [6] are the six-vertex model, and the quantum field theory of the massless sine-Gordon model. The Bethe ansatz, TQ relations and fusion hierarchies all have a rôle to play, and the works on these topics by Baxter [7], Klümper and Pearce [8], and Bazhanov, Lukyanov and Zamolodchikov [9-11] are particularly relevant.

In a short survey such as this, it is impossible to do justice to all of this background. In the next two sections some of the basic vocabulary will be sketched. More detailed reviews have been given in [12,13], and an extended version is currently in preparation. Subsequent developments following the initial observation in [6] can be found in [14-28].

\section{First prologue: the basics of integrable models}

Consider a rectangular two-dimensional lattice, of size $N \times M$. On each link of the lattice, attach an arrow, or spin, $\sigma$, pointing in one of the two possible directions along the link. An assignment of a spin to each link $i$ of the lattice gives a configuration $\left\{\sigma_{i}\right\}$. If we impose the additional constraint that the number of arrows pointing into each vertex is equal to the number pointing out, then we are on the way to defining the six-vertex model. The main task is to calculate averages over all possible configurations, weighted by relative probabilities which can be found as follows. First, the local Boltzmann weights $W[a, b, c, d]$ must be specified, one number for each possible set of values taken by the spins on the four links connected to a single site of the lattice. Then the relative probability of a given configuration is simply the product of these local weights over all sites. A key quantity is now the sum of these relative probabilities over all possible configurations, which gives the normalisation factor for the calculation of the actual probability of any quantity. Otherwise known as the partition function, it is given schematically as

$$
Z=\sum_{\left\{\sigma_{i}\right\}} \prod_{\text {sites }} W[a, b, c, d] .
$$

The local Boltzmann weights $W$, and hence also the partition function $Z$, may depend on physical parameters such as the temperature. For the six-vertex model there is just one physically-relevant quantity to specify, namely the anisotropy $\eta$. In the related quantum field theories this becomes the sine-Gordon coupling $\beta$, related to $\eta$ by $\beta^{2}=1-2 \eta / \pi$. For the solution technique we wish to describe below, however, a rather less physical parameter will be especially important, for reasons which will be sketched shortly: the spectral parameter $\xi$.

To evaluate $Z$, we can first define the transfer matrix $\mathbb{T}$, to be the sum over the spins on a line of horizontal links of the product of their Boltzmann weights, for a given set of 
spins on the adjacent vertical links above and below. If we assign one multiindex $\alpha$ to the vertical links above the line in question, and another $\alpha^{\prime}$ to the links below, then $\mathbb{T}$ can be thought of as a $2^{N} \times 2^{N}$ matrix $\mathbb{T}_{\alpha^{\prime}}^{\alpha}$, and, for periodic boundary conditions, the partition function $Z$ is

$$
Z=\operatorname{Trace}\left[\mathbb{T}^{M}\right]
$$

Clearly, if we can diagonalise $\mathbb{T}$, then we shall be able to compute $Z$. Even better, since the main interest is in the so-called thermodynamic limit when both $N$ and $M$ tend to infinity, we only need find the lowest-lying eigenvalues. Note that the mathematical structures found in this limit can also be obtained directly in the context of the continuum quantum field theory of the massless sine-Gordon model on a cylinder, using the constructions of [9-11].

Now comes the first moment where the concept of integrability arises: the local Boltzmann weights for the six-vertex model are so defined that the transfer matrices taken at different values of the spectral parameter commute:

$$
\left[\mathbb{T}(\xi), \mathbb{T}\left(\xi^{\prime}\right)\right]=0 .
$$

(The deeper meaning of this property would take us too far afield, but from one point of view it is related to the existence of infinitely-many commuting conserved quantities.) If the matrices $\mathbb{T}(\xi)$ all commute, then they can be diagonalised simultaneously and we can study individual eigenvalues $T(\xi)$ as functions of $\xi$. There are various methods for finding these functions, but the most relevant here was discovered by Baxter in the early 1970s, in connection with his work on the more-complicated eight-vertex model. Since the transfer matrix is an entire function of $\xi$ with $\xi$-independent eigenvectors, the eigenvalues $T(\xi)$ must also be entire. Baxter showed further that, for each eigenvalue $T(\xi)$, there is an associated function $Q(\xi)$, also entire, such that the following $T Q$ relation holds:

$$
T(\xi) Q(\xi)=e^{-2 \pi i p} Q\left(q^{-2} \xi\right)+e^{2 \pi i p} Q\left(q^{2} \xi\right)
$$

where the phase $q$ depends on $\beta$ as $q=e^{i \pi \beta^{2}}$, and the extra parameter $p$ allows for the possibility of inserting a 'twist' into the periodic boundary conditions on the lattice. (Strictly speaking, for finite values of the lattice width $N$ the TQ relation contains some extra factors, but these go in the thermodynamic limit. We are following the conventions of [10], appropriate for this limit, save that there $Q$ is denoted $A$ for $p \neq 0$, and both it and $T$ are taken to be entire functions of the squares of their arguments.) To see why this equation provides a useful constraint, we can argue as follows. Since $Q$ is entire, it is, subject to suitable growth conditions, determined by the location of its zeroes. Suppose these zeroes are at $\left\{e_{0} \ldots e_{k}\right\}$; then

$$
Q(\xi)=Q(0) \prod_{i=0}^{k}\left(1-\frac{\xi}{e_{i}}\right) .
$$

Now $T(\xi)$ is also entire, so the TQ relation (4) taken at $\xi=e_{j}$ implies that $e^{-2 \pi i p} Q\left(q^{-2} e_{j}\right)+$ $e^{2 \pi i p} Q\left(q^{2} e_{j}\right)=0$. Rearranging and using the product form (5),

$$
\prod_{i=0}^{k}\left(\frac{e_{i}-q^{2} e_{j}}{e_{i}-q^{-2} e_{j}}\right)=-e^{-4 \pi i p} .
$$


This gives us $k+1$ equations in $k+1$ unknowns, which determine the $e_{i}$ up to discrete ambiguities corresponding to the fact that the transfer matrix has not one but many eigenvalues. These equations coincide with the so-called Bethe ansatz equations (BAE) found in other, more direct, approaches to the problem. In this context, the $e_{i}$ are sometimes called the Bethe roots. Note, once the Bethe roots are known, the eigenvalue $T(\xi)$ is easily recovered by using (5) and then (4). Although the BAE (6) do not determine the Bethe roots uniquely, for the so-called ground state eigenvalue - the largest one, most important in the thermodynamic limit - this ambiguity is fixed by the fact that the Bethe roots for this eigenvalue are all located on the positive real axis.

Over the last few decades, Bethe ansatz equations such as (6) have been studied extensively, and much is known about their solutions. For the moment the key thing to remember is the simple way that they follow from the TQ relation. If a similar functional equation can be found in another context, we can hope to establish a rather detailed connection with the already-existing body of knowledge on integrable models.

\section{Second prologue: $\mathcal{P} \mathcal{T}$-symmetric quantum mechanics}

We now make a change of tack, and turn our attention to some quantum-mechanical problems first investigated by Bessis and Zinn-Justin in the early 1990s [3], and then successively generalised in $[4,5,17,24,25]$. For the current discussion it will suffice to consider the following collection of non-Hermitian 'position space' Hamiltonians:

$$
\mathcal{H}_{M, l}=-\frac{d^{2}}{d x^{2}}+(i x)^{2 M}+\frac{l(l+1)}{x^{2}},
$$

where $M$ is a positive real number. The interest is in the spectrum of $\mathcal{H}_{M, l}$ : those values of $E$ such that the ordinary differential equation $\mathcal{H}_{M, l} \psi=E \psi$ has a normalisable solution. By 'normalisable', for $M<2$ we simply mean square-integrable on a contour running along the real axis, distorted just below the origin to avoid the singularity there when $l(l+1) \neq 0$. For $M \geq 2$, the contour must be further distorted to ensure the correct analytic continuation, as explained in [4].

In figure 1 we show the spectrum as a function of $M$ for two negative values of $l$. Note that $M=1$ corresponds to the exactly-soluble simple harmonic oscillator with angular momentum, and that this point marks a profound change in the nature of the spectrum: for $M \geq 1$ it is entirely real, while for $M<1$ infinitely-many eigenvalues pair off and become complex. The reality of the spectrum for $M=3 / 2, l=0$ was the subject of Bessis and Zinn-Justin's original conjecture [3], which was extended to all $M \geq 1, l=0$ by Bender and Boettcher in [4]. However a complete proof was surprisingly elusive, and has only recently been given, making essential use of the ODE/IM correspondence [24].

The nature of the transition to complex eigenvalues as $M$ falls below 1 looks rather simple on figure 1a, but less so once figure $1 \mathrm{~b}$ has been examined. In fact, as shown in figure 2 , for $l \geq 0$ the connectivity pattern seen in figure 1a is completely reversed. Some further discussion of this behaviour can be found in [17,29], while the even-richer structure which emerges when a suitably-chosen inhomogeneous term is added to the potential is discussed in $[24,25]$.

As emphasised in $[4,5]$, a key feature of the Hamiltonians (7) is that, while not Hermitian, they are unchanged by the combined action of parity $\mathcal{P}$ and time-reversal $\mathcal{T}$ - whence the 


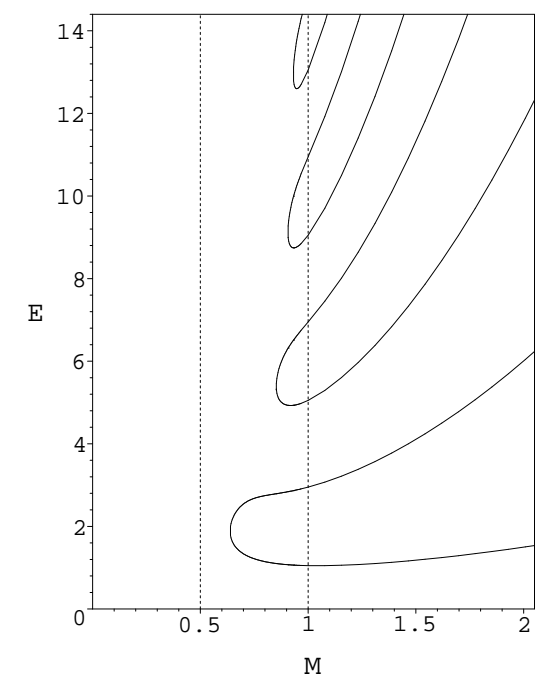

1a: $l=-0.025$

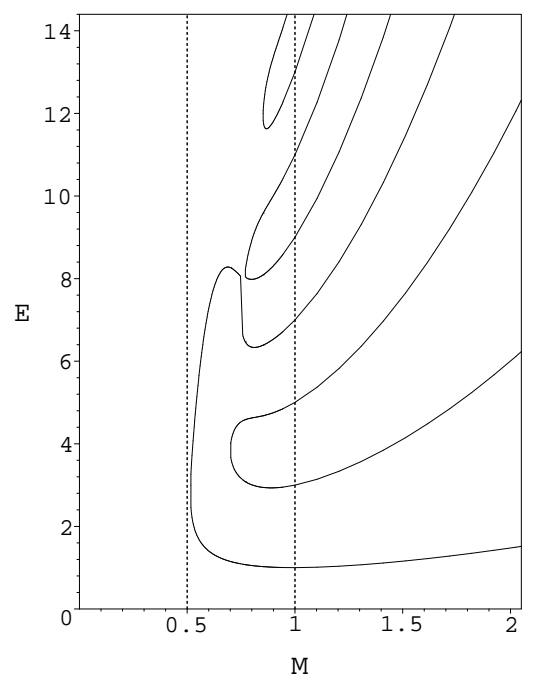

1b: $l=-0.001$

Figure 1. Eigenvalues of $\mathcal{H}_{M, l} \psi=E \psi$, plotted as a function of $M$ at $l=-0.025$ and $l=-0.001$.

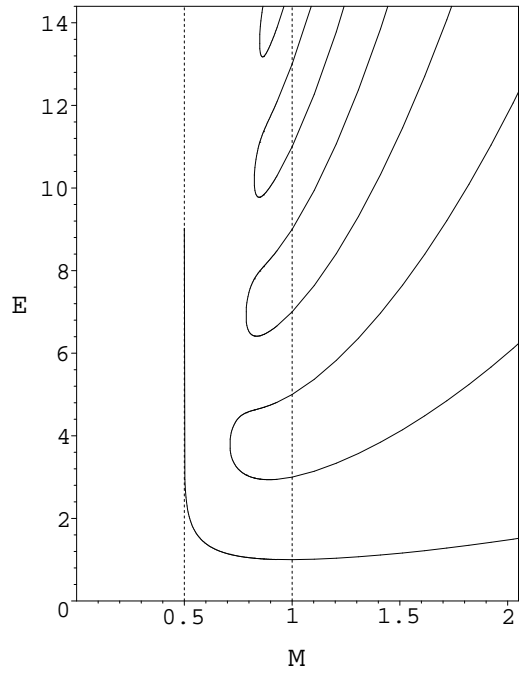

$2 \mathrm{a}: l=0$

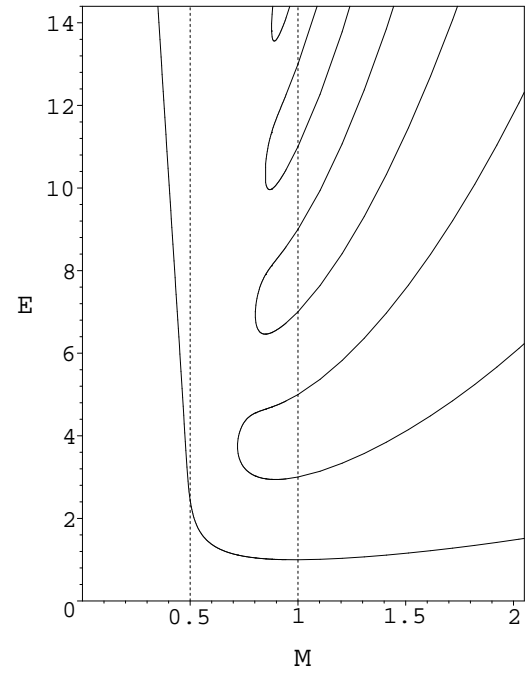

2b: $l=0.001$

Figure 2. Eigenvalues of $\mathcal{H}_{M, l} \psi=E \psi$, plotted as a function of $M$ at $l=0$ and $l=0.001$.

name $\mathcal{P} \mathcal{T}$-symmetric quantum mechanics. We hope to have demonstrated that the subject contains many interesting phenomena for the mathematical physicist to explore; see [30-49] and references therein for some further developments in the area. However, from the point of view of this article, its main interest lies in its surprising links with the six-vertex model discussed in section 2 above, which we shall now describe. 


\section{Spectral determinants and the correspondence}

Rather than worrying about individual eigenvalues of (7), it turns out to be useful to combine them into a single function - a spectral determinant - and then exploit the analytic properties of this function. This approach was particularly advocated in the differential equation context by Sibuya [1] and Voros [2]. Here, influenced by parallels with the theory of integrable models, we shall use spectral determinants to couple the non-Hermitian problem discussed so far with a related Hermitian problem, obtained from (7) by sending $x \rightarrow x / i$ and $E \rightarrow-E$. The new problem is

$$
\left(-\frac{d^{2}}{d x^{2}}+x^{2 M}+\frac{l(l+1)}{x^{2}}\right) \Phi(x)=E \Phi(x),
$$

and we choose as boundary conditions that the solution should vanish as $x \rightarrow \infty$ along the real axis, and behave as $x^{l+1}$ as $x \rightarrow 0$. For $\Re e l>-1 / 2$, this problem is Hermitian. In the language of ordinary differential equations in the complex domain, it is sometimes called a 'radial' problem, while (7) is called a 'lateral' problem. (Note, the radial problem can also be considered for $\Re e l \leq-1 / 2$, but is best then defined by analytic continuation in $l$.)

Let $\left\{E_{j}\right\}$ be the set of eigenvalues of $(7)$, and let $\left\{e_{i}\right\}$ be the eigenvalues of (8). Then define two spectral determinants, as follows:

$$
T(E)=T(0) \prod_{j=0}^{\infty}\left(1+\frac{E}{E_{j}}\right),
$$

and

$$
Q(E)=Q(0) \prod_{i=0}^{\infty}\left(1-\frac{E}{e_{i}}\right)
$$

Both products are convergent for $M>1$, and define entire functions of $E$, and their zeroes (or, for $T(E)$, their negatives) coincide with the eigenvalues of the corresponding spectral problem. For $M \leq 1$ convergence factors must be added, and it is more efficient to define the spectral determinants indirectly, via certain special 'Sibuya' solutions to (8), which are anyway needed to prove the key identity $(11)$ below; see $[1,17]$ for more details.

By considering the asymptotic behaviour of the Sibuya solutions in the complex plane it is not hard, following the arguments given in [17], to establish a Stokes relation, from which one can obtain the following functional equation

$$
T(E) Q(E)=\omega^{-(2 l+1) / 2} Q\left(\omega^{-2} E\right)+\omega^{(2 l+1) / 2} Q\left(\omega^{2} E\right),
$$

where $\omega=e^{i \pi /(M+1)}$. This shows that the spectral problems (7) and (8) are related by much more than a simple change of variables and boundary conditions; and furthermore, that this relation is encoded precisely in a TQ relation of the sort which had previously arisen in the context of the six-vertex and sine-Gordon models.

\section{Consequences of the correspondence}

If we set $\beta^{2}=1 /(M+1)$ and $p=(2 l+1) /(4 M+4)$, then the two TQ relations (4) and (11) match perfectly. The remaining ambiguity in the solutions of the resulting Bethe ansatz equations is resolved once we recall that the problem (8) is Hermitian, and so all 
of its eigenvalues $e_{i}$ are real. Given the remarks about the locations of Bethe roots at the end of section 2, this means that the spectral determinant $Q(E)$ arising in the ordinary differential equation should be identified with the Q-function $Q(\xi)$ for the ground state of the sine-Gordon model. (We must also match the normalisations of $E$ and $\xi$, since these are left undetermined by the TQ relations. This is easily achieved by comparing the leading asymptotic of the field theory Q-function given in [10] with that obtained by a simple WKB analysis of the ordinary differential equation - see $[6,17]$ for more details.) Once the Qfunctions have been identified, so can be the T-functions, and we have our main punchline:

The spectral determinant $T$ for the lateral $(\mathcal{P} \mathcal{T}$-symmetric) problem $(7)$ is equal to the ground state, or vacuum, eigenvalue of the transfer matrix $\mathbb{T}$ of the massless sine-Gordon model on a cylinder.

Together with the corresponding statement for $Q$, this provides a powerful tool to analyse the functions constructed in the context of integrable quantum field theory in [9-11], as the analytic properties of solutions of ordinary differential equations are relatively easy to control. (In this regard, we should mention that, for a complete proof of the correspondence, it currently seems best to argue via the so-called 'quantum Wronskian' relation, as in $[6,14]$. However the route we have adopted here is perhaps more intuitive, and it leads naturally on to the reality proof which we shall describe shortly.) There are also less direct applications of the correspondence - for example, it yields a proof [14] of a duality relation in quantum Brownian motion, first proposed in [50]. In finishing this contribution, we mention two more, both on the ordinary differential equations side of the correspondence.

The first concerns the reality properties of the $\mathcal{P} \mathcal{T}$-symmetric problems (7). We have already seen, in section 2, the argument which shows that substituting $E=e_{j}$ into the TQ relation (11) leads to Bethe ansatz equations, which in the light of the correspondence we can reinterpret as coupling the different eigenvalues of the Hermitian problem (8). However, if we instead set $E=-E_{j}$, the same reasoning leads, in the region $M>1$ for which the factorised form (10) applies, to

$$
\prod_{i=0}^{\infty}\left(\frac{e_{i}+\omega^{2} E_{j}}{e_{i}+\omega^{-2} E_{j}}\right)=-\omega^{-2 l-1}
$$

This equation couples the so-far mysterious eigenvalue $E_{j}$ of the $\mathcal{P} \mathcal{T}$-symmetric problem (7) to the much better-controlled, and indeed real and positive, eigenvalues $e_{i}$ of the Hermitian problem (8). If we take the modulus ${ }^{2}$ of both sides, it is soon seen that the only way that the resulting equality can be achieved is for $E_{j}$ to be real, as had previously been conjectured. Notice, the proof breaks down for $M \leq 1$, as has to be the case given the numerical findings of $[4,17]$, illustrated in figures 1 and 2 above. More details, in a slightly more general setting than that described here, can be found in [24]; for a further generalisation of the method, see [51].

The second application was also found in [24], but is a little more specialised. We consider the radial problem at $M=3$, and add an inhomogeneous term $\alpha x^{2}$ to the potential. Although this was not done in the original paper, it will also be convenient to reparametrise 
the angular-momentum term by setting $\lambda=\sqrt{3}(2 l+1)$, so that the equation becomes

$$
\left(-\frac{d^{2}}{d x^{2}}+x^{6}+\alpha x^{2}+\frac{\lambda^{2}-3}{12 x^{2}}\right) \Phi(x)=E \Phi(x) .
$$

Via the Bethe ansatz approach, it turns out that this problem has a relationship with a third-order ordinary differential equation:

$$
\left(D\left(g_{2}-2\right) D\left(g_{1}-1\right) D\left(g_{0}\right)+x^{3}\right) \phi=\frac{3 \sqrt{3}}{4} E \phi
$$

where $D(g) \equiv\left(\frac{d}{d x}-\frac{g}{x}\right)$, and

$$
g_{0}=1+(\alpha+\sqrt{3} \lambda) / 4, \quad g_{1}=1+\alpha / 2 \quad, \quad g_{2}=1+(\alpha-\sqrt{3} \lambda) / 4 .
$$

This third-order equation is associated with $S U(3)$ Bethe ansatz equations, as discussed in $[18,21]$. Furthermore, the third-order equation is symmetrical in $\left\{g_{0}, g_{1}, g_{2}\right\}$, a feature which is completely hidden in the original second-order equation. By playing with this symmetry, one can establish some novel spectral equivalences between different (secondorder) radial problems, and also between these and certain lateral problems. More details and explicit formulae can be found in [24], and here we simply add to that paper the remark that, when expressed in terms of the variables $(\alpha, \lambda)$, the mappings turn out to act as certain $2 \times 2$ matrices in the Weyl group of $S U(3)$.

Other aspects of the ODE/IM correspondence are still being developed as this contribution is being written. It would be nice to have a working correspondence also for finite lattice systems, before the thermodynamic limit is taken, and some small progress in this direction will be reported in [52]. Another important question that until recently remained open concerns the other states in the quantum field theories, besides the ground state, and whether they can also be matched with differential equations. In a very recent paper [53], Bazhanov, Lukyanov and Zamolodchikov have answered this in the affirmative. The 'Qpotentials' that they construct are no longer real, even for the radial problems. This is not as surprising as it might seem - recall that it is only for the ground state that the Bethe roots, which correspond to the eigenvalues of the radial spectral problem, all lie on the real axis. For other states there are complex roots, and so even the radial problems can no longer be Hermitian.

Finally, it would be valuable to have a more physical understanding of the relationship between integrable quantum field theories and ordinary differential equations. At the moment this remains mysterious, but sufficiently-many examples of the phenomenon have now been collected that progress may not be too far off.

\section{Acknowledgments}

PED thanks the organisers for the invitation to speak at the conference; TCD, AM-S and RT thank the UK EPSRC for a Research Fellowship, a Studentship and an Advanced Fellowship respectively. This work was partially supported by the EC network "EUCLID", contract number HPRN-CT-2002-00325. 


\section{References}

1. Y. Sibuya, Global Theory of a second-order linear ordinary differential equation with a polynomial coefficient (Amsterdam: North-Holland 1975).

2. A. Voros, J. Physique Lett. 43, L1 (1982); Ann. Inst. Henri Poincaré XXXIX, 211 (1983); J. Phys. A: Math. Gen. 32, 5993 (1999); Corrigendum J. Phys. A: Math. Gen. 34, 5783 (2000).

3. D. Bessis and J. Zinn-Justin, unpublished, circa 1992.

4. C.M. Bender and S. Boettcher, Phys. Rev. Lett. 80, 4243 (1998).

5. C.M. Bender, S. Boettcher and P.N. Meissinger, J. Math. Phys. 40, 2201 (1999).

6. P. Dorey and R. Tateo, J. Phys. A: Math. Gen. 32, L419 (1999).

7. R.J. Baxter, Ann. Phys. 76, 1 (1973); Ann. Phys. 76, 25 (1973); Ann. Phys. 76, 48 (1973).

8. A. Klümper and P.A. Pearce, J. Stat. Phys. 64, 13 (1991).

9. V.V. Bazhanov, S.L. Lukyanov and A.B. Zamolodchikov, Commun. Math. Phys. 177, 381 (1996).

10. V.V. Bazhanov, S.L. Lukyanov and A.B. Zamolodchikov, Commun. Math. Phys. 190, 247 (1997).

11. V.V. Bazhanov, S.L. Lukyanov and A.B. Zamolodchikov, Commun. Math. Phys. 200, 297 (1999).

12. P. Dorey, C. Dunning and R. Tateo, JHEP Proceedings PRHEP-tmr 2000/034, Nonperturbative Quantum Effects 2000, hep-th/0010148.

13. P. Dorey, C. Dunning and R. Tateo, Proceedings of the Johns Hopkins workshop on current problems in particle theory 24, Budapest, 2000 (World Scientific 2001).

14. V.V. Bazhanov, S.L. Lukyanov and A.B. Zamolodchikov, J. Stat. Phys. 102, 567 (2001).

15. J. Suzuki, J. Phys. A: Math. Gen. 32, L183 (1999).

16. P. Fendley, Lett. Math. Phys. 49, 229 (1999).

17. P. Dorey and R. Tateo, Nucl. Phys. B563, 573 (1999).

18. P. Dorey and R. Tateo, Nucl. Phys. B571, 583 (2000).

19. J. Suzuki, J. Phys. A: Math. Gen. 33, 3507 (2000).

20. J. Suzuki, J. Stat. Phys. 102, 1029 (2001).

21. P. Dorey, C. Dunning and R. Tateo, J. Phys. A: Math. Gen. 33, 8427 (2000).

22. J. Suzuki, Stokes multipliers, Spectral Determinants and T-Q relations, nlin-si/0009006.

23. K. Hikami, Nucl. Phys. B604, 580 (2001).

24. P. Dorey, C. Dunning and R. Tateo, J. Phys. A: Math. Gen. 34, 5679 (2001).

25. P. Dorey, C. Dunning and R. Tateo, J. Phys. A: Math. Gen. 34, L391 (2001).

26. V.V. Bazhanov, A.N. Hibberd and S.M. Khoroshkin, Integrable structure of $W_{3}$ Conformal Field Theory, Quantum Boussinesq Theory and Boundary Affine Toda Theory, hep-th/0105177.

27. V.V. Bazhanov, S.L. Lukyanov and A.M. Tsvelik, Analytical results for the Coqblin-Schrieffer model with generalized magnetic fields, cond-mat/0305237.

28. S.L. Lukyanov and A.B. Zamolodchikov, Integrable circular brane model and Coulomb charging at large conduction, hep-th/0306188.

29. P. Dorey, A. Millican-Slater and R. Tateo, in preparation.

30. E. Delabaere and F. Pham, Phys. Lett. A250, 25 (1998).

31. M. Znojil, Phys. Lett. A259, 220 (1999).

32. G.A. Mezincescu, J. Phys. A: Math. Gen. 33, 4911 (2000).

33. M. Znojil, J. Phys. A: Math. Gen. 33, 6825 (2000).

34. E. Delabaere and D. Trinh, J. Phys. A: Math. Gen. 33, 8771 (2000).

35. B. Bagchi, F. Cannata and C. Quesne, Phys. Lett. A269, 79 (2000).

36. C.M. Bender, M. Berry, P.N. Meisinger, Van. M. Savage and M. Simsek, J. Phys. A: Math. Gen. 34, L31 (2001).

37. C.M. Bender and Q. Wang, J. Phys. A: Math. Gen. 34, 3325 (2001).

38. G.A. Mezincescu, J. Phys. A: Math. Gen. 34, 3329 (2001).

39. C.R. Handy and X.Q. Wang, J. Phys. A: Math. Gen. 34, 8297 (2001).

40. V.M. Tkachuk and T.V. Fityo, J. Phys. A: Math. Gen. 34, 8673 (2001). 
41. C.M. Bender, S. Boettcher, H.F. Jones, P. Meisinger and M. Simsek, Phys. Lett. A291, 197 (2001).

42. F. Cannata, M. Ioffe, R. Roychoudhury and P. Roy, Phys. Lett. A281, 305 (2001).

43. G.S. Japaridze, J. Phys. A: Math. Gen. 35, 1709 (2002).

44. R. Kretschmer and L. Szymanowski, The Interpretation of Quantum-Mechanical Models with Non-Hermitian Hamiltonians and Real Spectra, quant-ph/0105054.

45. A. Mostafazadeh, J. Math. Phys. 43, 205 (2002).

46. C.M. Bender, D.C. Brody and H.F. Jones, Phys. Rev. Lett. 89, 270401 (2002).

47. A. Mostafazadeh, J. Math. Phys. 44, 974 (2003).

48. C.M. Bender, P.N. Meisinger and Q. Wang, J. Phys. A: Math. Gen. 36, 1973 (2003).

49. S. Weigert, Completeness and Orthonormality in PT-symmetric Quantum Systems, quant-ph/0306040.

50. M.P.A. Fisher and W. Zwerger, Phys. Rev. B32, 6190 (1985).

51. K.C. Shin, Commun. Math. Phys. 229, 543 (2002).

52. P. Dorey, J. Suzuki and R. Tateo, Finite lattice Bethe ansatz systems and the Heun equation, hep-th/0308053.

53. V.V. Bazhanov, S.L. Lukyanov and A.B. Zamolodchikov, Higher-level eigenvalues of $Q-$ operators and Schrödinger equation, hep-th/0307108. 